\title{
Determinants of Farmers' Risk Perceptions of Hailstorms in Northern Bangladesh: Is Adaptive Capacity the Major Concern?
}

\author{
Md Lamiur Raihan*, Mrittika Basu, Kenichiro Onitsuka, Satoshi Hoshino \\ Laboratory of Sustainable Rural Development, Graduate School of Global Environmental Studies, \\ Kyoto University, Kyoto 606-8502, Japan
}

Received: 4 November 2020

Accepted: 10 April 2021

\begin{abstract}
Extreme weather events (EWEs) such as hailstorms are an increasing threat to agriculture worldwide. To develop successful and efficient hailstorm risk management policies, it is important to understand the composition of farmers' hailstorm risk perceptions. This study aims to identify the determinants of these risk perceptions. This is done by collecting pertinent information on the perceptions of risk, farm traits, adaptive capacity, and various other personal characteristics from farmers' households in hailstorm-oriented areas of northern Bangladesh. By analyzing the explanatory power of various risk factors using the binary logistic regression model, this study reveals that the hailstorm risk perceptions of farmers are embodied by subjective risk factors, such as their perceived control of hailstorm risk, and their personal circumstances. Their perceived resources for tackling hailstorm risk are the most significant predictor variable of hailstorm risk perceptions. Marginal and smallholder farmers are very vulnerable and perceive more risk than is the case with the medium-holder and large-holder farmers, owing to the lack of a compensation policy after the occurrence of hailstorms. The relevant authorities should pay urgent attention to managing hailstorm risks by developing adequate adaptive capacity among farmers in northern Bangladesh.
\end{abstract}

Keywords: hailstorms, risk perception, Bangladesh, binary logistic regression, adaptive capacity

\section{Introduction}

The world is facing a multitude of climate change and environmental issues. Various studies suggest that climate-related events and natural hazards are increasing worldwide [1-3]. EWEs, such as droughts, thunderstorms, hailstorms, and lightning, are increasing

*e-mail: raihan.lamiur.33m@st.kyoto-u.ac.jp on a daily basis [4] with impacts that are more visible [5]. Countries with developing economies are more vulnerable to the continuously changing climate, owing to the huge impact on their agricultural sectors caused by the increasing frequency of EWEs and disasters [6]. Over the past decade, developing economy-based countries have faced annual losses of United States dollars (USD) 35 billion caused by various natural disasters and, more specifically, EWEs [3]. These losses in the economic sector have strong linkages in both 
forward and backward directions between and within socio-economic conditions [7-9].

Hailstorms are natural hazards that are generally over a small scale; however, regardless of their localized occurrence, they are considered a major natural hazard that leads to huge economic losses to the agricultural sector, property, and livelihoods [5]. Most of the related existing research demonstrates the climatology of thunderstorms and hailstorms [10-11], their causes and consequences [10], and coping strategies [12]. Raihan et al. (2020) studied the emergence of hailstorm risk and its potential threats to sustainable agriculture in Bangladesh [13]. Understanding worldwide was found to be inadequate in relation to the community risk analysis of hailstorms, farmers' adaptive capacity, and identification of the determinants of risk perceptions regarding hailstorms.

Bangladesh is very vulnerable to climate change due to its geographical location and is considered to be one of the most natural hazard-susceptible countries in the world [14-15]. It is regularly affected by tidal floods and flash floods, cyclones, coastal erosion, storm surges, soil salinity, thunderstorms with or without hail, droughts, and lightning that cause an enormous loss of life and property and, very importantly, significantly affect agricultural productivity [16]. The severity of climate change and the increase in EWEs ultimately result in harmful impacts on Bangladesh's largest gross domestic product (GDP) earning sector, that is, the agricultural sector [17]. Rural communities have developed some coping and adaptive strategies for conventional hazards, such as droughts, floods, soil salinity, and cyclones, and the government has a well-established policy to support victims [16], whereas no explicit policy has been established to address the issue of hailstorms [13]. However, for most smallholder farmers in Bangladesh, the successful adoption of different adaptation strategies and the development of adaptive capacity at the individual farmer's level as well as at the community level are very important to lessen their vulnerability and reduce the potential risks of all natural hazards faced [18], with hailstorms no exception.

Accurate risk perception is indispensable when adopting a high-yielding risk management strategy as it is believed that the farmer who is unaware of the risk confronted is unable to manage it practically [19-20]. Effective adaptation measures to minimize the risks to the agricultural sector rely on risk perception at the individual level, the ability to control the risks, and supports or incentives from the relevant authority after a catastrophic hazard occurrence [21]. Several existing studies have shown positive relationships between natural hazard risk perceptions and adaptive capacity as well as decision making for adaptation behaviour $[18,22,23,24]$. With all other natural hazards and, in this case, hailstorms, understanding farmers' hailstorm risk perceptions is also important as this could form the basis for understanding their potential responses to hailstorm risk management. Various studies have inquired about farmers' risk perceptions and the determinants in relation to climate change as a whole [24], as well as for individual hazards such as floods [25], droughts [7], cyclones, earthquakes [26], etc. but the focus on risk factors for hailstorms is almost nil. Moreover, hailstorms are the most prioritized natural hazard in northern Bangladesh when considering the vulnerability of its sustainable agriculture [13]. Northern Bangladesh is seriously affected by hailstorms, resulting in a significant impact on the agricultural sector and rural livelihoods [27]. As hailstorms are acknowledged as potential hazards in northern Bangladesh [13], it is necessary to be acquainted with the different factors that act as determinants for hailstorm risk perceptions so these hazards can be better managed.

This research aims to measure the determinants which are the most important contributors to farmers' hailstorm risk perceptions. Assessment of these determinants will be helpful in improving the adaptive capacity of farming communities. Moreover, this could assist scholars and, most importantly, policy makers when designing effective hailstorm risk management policies. This study will also enrich the very limited existing literature on the determinants that affect farmers' hailstorm risk.

\section{Literature Review and Construction of the Conceptual Framework}

Today, risk perception is widely used terminology in climate change risk management and adaptation, the entrepreneurship decision-making process [28], and policy making [29]. According to Sjoberg (1998), the perception of risk is a subjective judgement of the likelihood of a respective event such as flood, drought, cyclone, etc. and stakeholders' subsequent awareness of its level of damage [30]. The three approaches used to measure the risk are objective, subjective, and perceptive [31], with none of these approaches correct on its own as, using a single approach to solve one risk, is not possible [32]. In the objective approach, based on expected utility theory, it is assumed that stakeholders have sufficient information on the probabilities and consequences regarding a risky event [23], with this theory widely used to investigate different extreme weather events, disaster-induced losses, and adaptation strategies in the agricultural sector. This theory suggests that, as farmers have sufficient information on any natural hazard's probabilities and impacts, their risk perceptions are in line with the original risk and can be perfectly assessed using objective risk factors [33]. However, most people rely on potential negative consequences to measure risks [24]. Duinen et al. (2015) defined objective risk factors are those that determine a stakeholder's original risk [7]. He used two objective risk factors, namely, "exposure" and "sensitivity," the same factors used by Patt and Schroter (2008) [34], Roehl and Fesenmaier (1992) [35], Keil et al. (2000) 
[36], Nosic and Webber (2009) [29], and Hasan and Kumar (2019) [37]. The term 'exposure' indicates the perceived probability of the occurrence of the hazard, while the term 'sensitivity' relates to its overall negative consequences [37].

In contrast to the objective approach, the subjective approach asserts that risk is not always exclusively objective: rather, it differs based on the stakeholder's perception which is influenced by his/her knowledge, skills, and experiences; perceived control over the risk; social norms; and personal status. Finally, the perceptive approach to risk is known as the sum of all negative impacts that should be plausible to an individual who has experience of the variations of frequency, impact, and effects [31]. Pat and Schroter (2008) showed that behavior-related factors affect farmers' risk perceptions to climate change which vary from the expert estimation of risk [34]. Previous studies on factors that determine risk perception at different levels demonstrated that farmers' perceptions are very much determined by their socio-economic characteristics and their farms' features [38-40]. The differences in risk perception owing to different socio-economic characteristics are evident [41], with variations found across different farming groups [9]. Moreover, several studies included sociodemographic characteristics as predictor variables of risk perceptions of hazards [42]. Different studies have shown different directions in the relationship between socio-demographic variables and risk perceptions to natural hazards at different levels; thus, no agreed model has been established for the relationship between socio-demographic and economic factors, and perceived risk [43]. However, based on the literature survey and experts' views, the socio-demographic and economic characteristics should be selected for further study.

Several studies on risk perceptions revealed that the perceived ability to control the risk is negatively related to the individual stakeholder's own risk perceptions [44]. The perceived feeling of ability to tackle the specified risks indicates the perceived capability to protect oneself against those risks [30]. Perceived ability can also be defined as farmers' perceptions of their capacity to adjust to a specific risk, that is, adaptation efficacy; to minimize potential damage using their self-skills and knowledge; to cope with the consequences, recognized as self-efficacy; and to have the resources to manage the risks involved, also known as adaptation costs [37].

Duinen et al. (2015) measured subjective risk factors as a set of the individual stakeholder's personal traits or socio-demographic and economic variables and the ability to control the risk, that is, the psychological variables [7]. These authors also included social influence as a factor in the risk perception of drought. However, in our research, we avoided social influence as the sole risk factor of hailstorm risk perception; instead, it was considered under policy factors as it is actually an external factor. The compensation policy and support or incentives system after the occurrence of any natural hazard play an important role in risk management. Evaluation of insurance losses and the provision of financial support based on the insurance model in the case of hailstorm occurrences and losses to the agriculture sector and property are very common worldwide [45]. However, in Bangladesh, the government has no explicit policy to address the hailstorm risk management issue. Moreover,

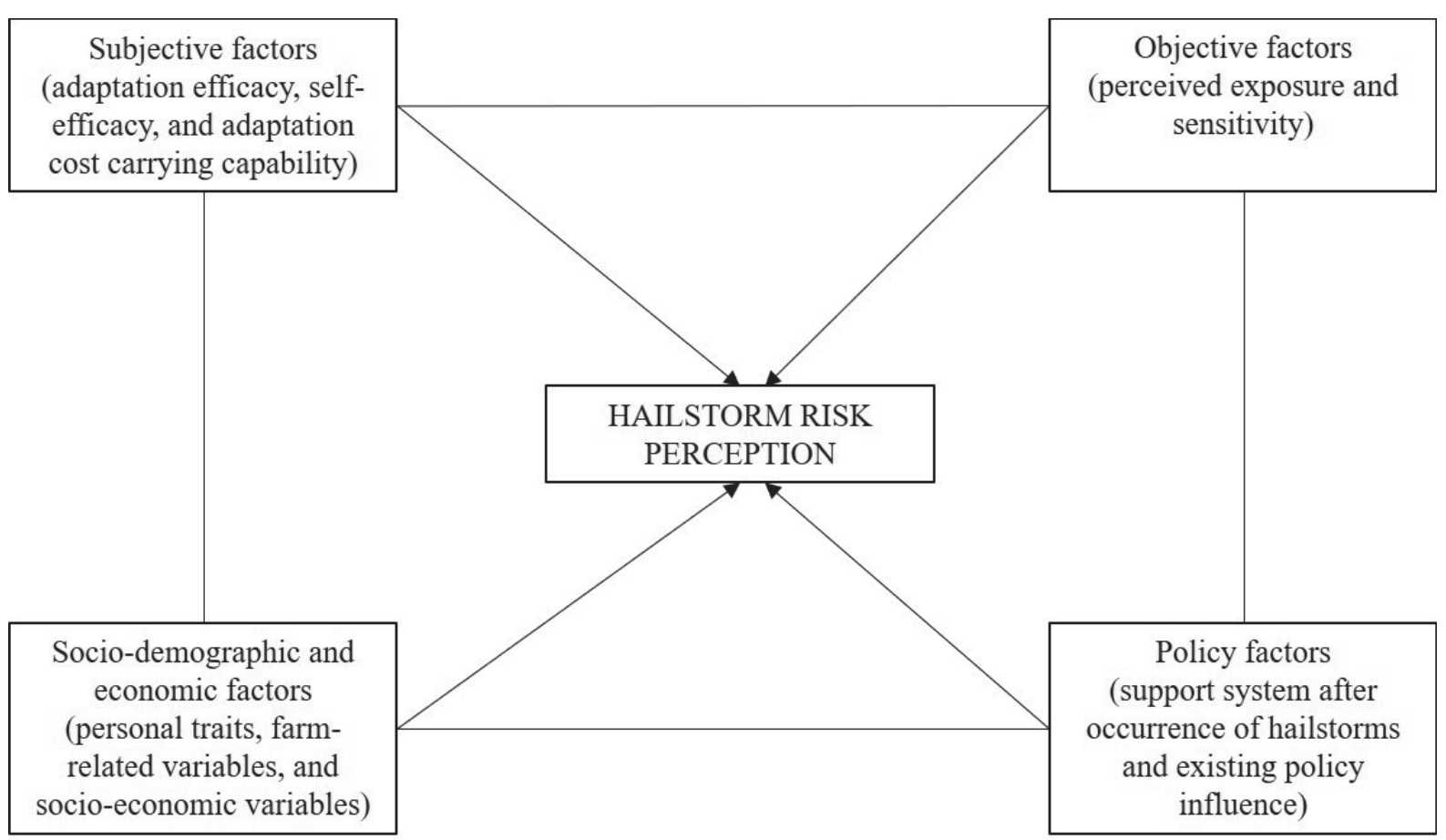

Fig. 1. Conceptual framework of the study. 
Raihan et al. (2020) found that, owing to the lack of a strong policy, farmers are losing their motivation for farming and are perceiving increased risks [13]. Thus, our study included farmers' perceptions of the policies of the government and non-governmental organizations (NGOs) in controlling hailstorm risk as a risk perception factor and modified the framework developed by Duinen et al. (2015) [7] for use in our research. The study defines hailstorm risk perceptions as concerns shown by farmers regarding previous, present, and future incidents of negative impacts on crop production and agricultural activities due to the occurrence of hailstorms.

\section{Methodology}

\section{Features of the Study Area}

This study focuses on farmers in the Panchagarh district of Bangladesh which is lying between latitudes $26^{\circ} 17^{\prime}$ and $26^{\circ} 29^{\prime}$ north and between longitudes $88^{\circ} 31^{\prime}$ and $88^{\circ} 46^{\prime}$ east. Fifty-six villages under the two Union council areas of Haribhasa and Hafizabad were chosen as the study area. This region is among the regions that are very important in Bangladesh for crop production owing to its diversified weather pattern, diversity of crops cultivated, variations in field crop cultivation, big orchards, and high cropping intensity (205\%) [46]. Most people rely on agriculture and, throughout the year, face different natural hazards [46]. These two Union council areas were selected based on the Bangladesh Department of Agricultural Extension's reports, and consultations with local agricultural department officials and resources personnel on the significance of crop production and the natural hazard profile. Every year, severe agricultural droughts occur in Panchagarh and, as a result, $30-40 \%$ of crop loss occurs due to the scarcity of surface water and limited irrigation facilities [47]. Hailstorms occur every year, and about $55-75 \%$ of crops are totally destroyed after a catastrophic storm; thus, this district is prioritized as facing the riskiest hazards in the region [13]. The map of the study area is shown in Fig. 2.

\section{Survey Design and Data Collection}

This study collected primary data through the survey conducted from April-May 2019. Data were collected from farmer households using a structured questionnaire. The study area had 1,925 farming households (population), with 320 being the sample size for the survey (using the survey system calculator, at 95\% confidence interval [CI]) [13]. Farmers were chosen using simple random sampling from the list collected from the district agriculture office. The questionnaire comprised questions related to a wide range of variables, including socio-demographic characteristics, farm size, economic variables, hailstorm objective and subjective risk variables, and perception of risk feelings towards hailstorms. The socio-demographic variables included were the farmer's age and education, while the farm-related variables were farm size and predecessor farm size. Socio-economic variables comprised annual family income; household assets (total assets as a monetary amount); credit received (from both formal and informal sources); training experience (number of days attended); organizational affiliation (4-point rating scale for the categories of executive, executive committee member, ordinary member, or not at all); and their extension media contact for access to information [37]. To measure their extension media contact for farmrelated information, a 4-point rating scale was used to assess how frequently (not at all, rarely, occasionally, frequently) a farmer accessed the 12 different farm information sources. The media sources were: local leader; local agriculture officer, known as Sub-Assistant Agriculture Officer (SAAO) or Upazila Agriculture Officer (UAO) or Agriculture Extension Officer (AEO); model farmer; Water Development Board officer; input dealer; participation in group meetings; method demonstrations and results demonstrations; agricultural fairs; listening to the agricultural program on radio; watching the agricultural program on television; reading leaflets/posters/bulletins/newspapers; and using a mobile phone or the internet to search for agriculturerelated information [37]. For the convenience of respondents, all questions were translated into the study area's local language.

\section{Dependent Variable}

The dependent variable of this study was farmers' risk feelings towards hailstorms. In the first step of the data collection, farmers were asked to reply to one broad question: have you perceived any sort of risk to your crop production and agricultural activities due to hailstorm occurrences? The binary responses (yes/no) were recorded and used in the empirical analysis. In the second step, respondents who answered 'yes' to the first question were asked to rate their level of hailstorm risk perception based on a 4-point rating scale (i.e., 'highly perceived,' 'moderately perceived,' 'less perceived,' and 'not sure') [41]. This question in the second step was asked to gain a better understanding of each respondent's risk perception but it had no use in the empirical analysis.

\section{Analysis of Determinants of Hailstorm Risk Perception}

Binary logistic regression is a predictive analysis which is used to predict the likelihood that a given observation, based on one or more dependent variables that can be either continuous or categorical, falls into one of two types of a dichotomous dependent variable [48]. Linear regression estimates the value that $Y$ takes, but the frequencies of values 0 and 1 are used in logistic 
regression to estimate the likelihood of $Y$ taking a particular value (Eq. 1) [48].

$$
P(Y)=\frac{e^{b o^{+b_{1} x_{1}+b_{2} x_{2}+\cdots}+b_{n} x_{n}}}{1+e^{b o^{+b_{1} x_{1}+b_{2} x_{2}+\cdots}+b_{n} x_{n}}}
$$

$P$ : probability of $Y$ occurring

$e$ : natural logarithm base

$b_{0}$ : interception at the y-axis, a constant term

$b_{1}:$ line gradient

$b_{n}$ : regression co-efficient of $X_{n}$

$X_{1}^{n}$ : predictor variable $X_{1}$ predicts the probability of $Y$.

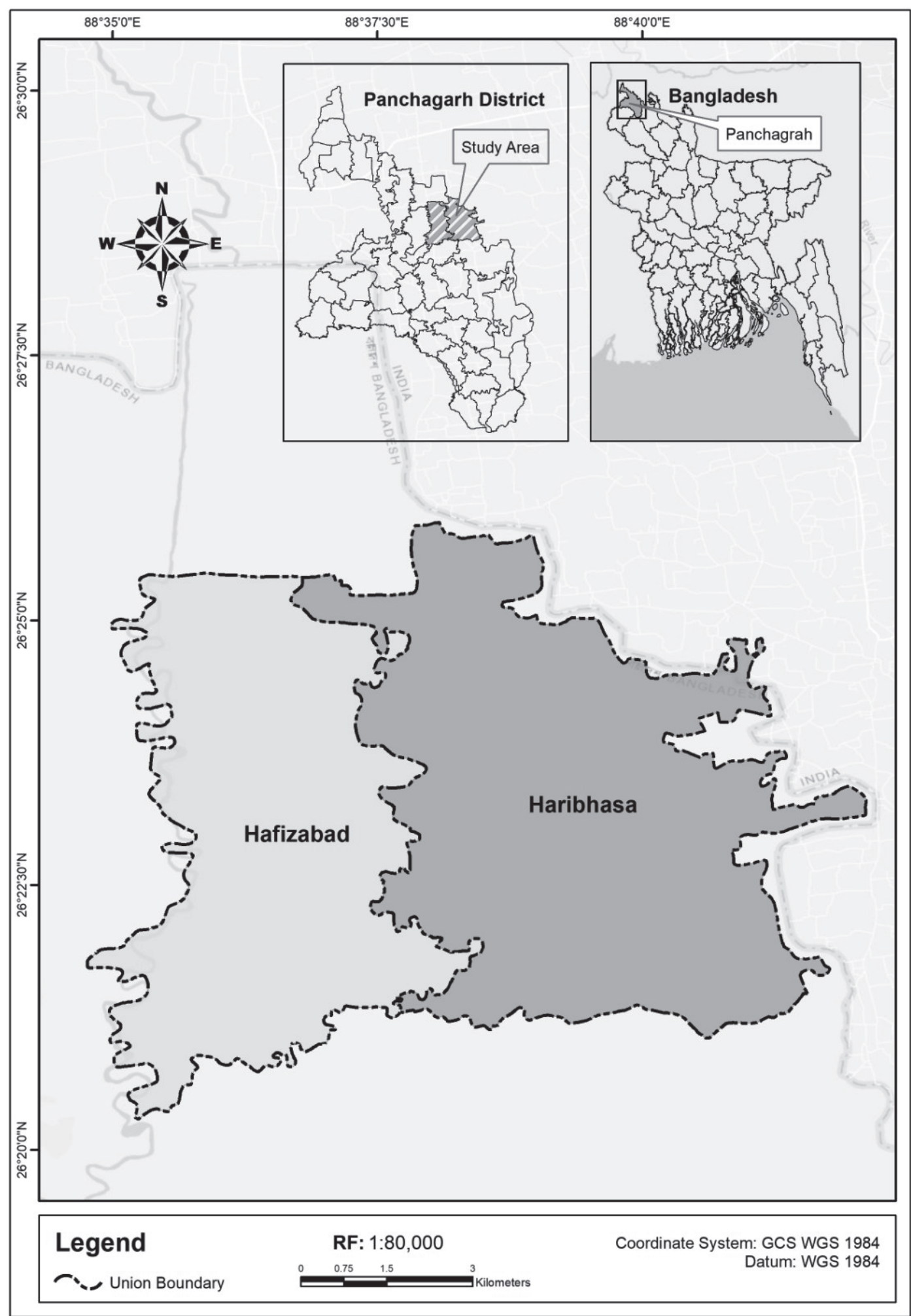

Fig. 2. Map of the study area [13]. 
The above logit model was used to assess the contribution of socio-economic factors, objective risk factors, subjective risk factors, and policy influence in affecting farmers' risk perceptions of hailstorms. The nature of the dependent variable was binary; therefore, neither the classical linear regression model (CLRM) nor the simple linear probability model (LPM) were suitable for this study [33]. Before running the model, the bivariate Pearson correlation coefficient test was executed to check the correlations between independent variables to avoid predictor variables that are highly dependent and strongly correlated with each other, as well as to check their relationships with the dependent variable (e.g., risk perception) [49].

In this study, the independent variables for hailstorm risk perception were classified into four categories: socio-demographic and economic factors (age, level of education, farm size, predecessor farm size, annual family income, household assets, agricultural training experience, credit received, extension media contact, and organizational participation); objective risk factors (exposure and sensitivity to hailstorms); and subjective risk factors (adaptation efficacy, self-efficacy, and adaptation cost). For policy influencing factors, respondents were meant to be asked two questions. Whether they perceived the existence of any policy to provide support after a hailstorm's occurrence (a perception factor) was the first question, while the second question was: did they receive any incentives or support after a catastrophic hailstorm had occurred? As all respondents answered that they did not perceive that any policy was available to support them, the second question was not asked. Respondents were asked to score their responses on each of the risk factors and adaptive capacity factors using a 5-point scale (i.e., 'very high,' 'high,' 'medium,' 'low,' and 'very low') $[37,50]$. In this study, we used a dichotomous dummy variable for the objective and subjective risk factors [50]. The 5-point rating scale was further coded into binary responses such as 'high' and 'low.' The positive responses ('very high,' 'high,' and 'medium') were scored 1, and the negative responses ('low' and 'very low) were given a score of 0 (Table 1) [51]. The model could not include both objective risk factors and policy factors as the data for these variables were not normally distributed. The data were analyzed by IBM SPSS Statistics package 25.0.

\section{Results}

\section{Socio-Demographic Characteristics of Farmers and Their Risk Perceptions}

The salient features of the respondent farmers' socio-economic and demographic characteristics are shown in Table 1. The respondents were dominated by age groups around middle-age (mean $=49.9$ years with standard deviation $[\mathrm{SD}]=13.48$ years). The respondents had mostly primary or secondary levels of education. The largest percentage of the farmers had small-sized farms. However, this was different for their predecessor farm size. Most respondents predecessor had mediumsized or large farms or had their own land. The average annual income of the respondent farmers was USD 1,300, with the largest percentage of the farmers having low or medium income. Most farmers had low household assets, with the mean value being USD 1,450. The largest percentage of the farmers only had a short duration of agricultural training exposure, with the average being almost four days in a year.

Almost all respondent farmers obtained credit from various banks and NGOs for crop production and farm management. The average amount of credit was USD 240 in the cropping season. The largest percentage of the farmers had medium-level information access to various extension media sources with mediumlevel participation in various agricultural and social organizations. In all, 94\% of the farmers perceived a high probability of occurrence (exposure) of hailstorms and almost 95\% perceived a high impact (sensitivity) of hailstorms on agricultural and crop production. Moreover, the psychological factors, known as adaptive capacity in this study, in general reflected a scenario of low adaptive capacity. Most farmers perceived that they had low adaptation efficacy, and low knowledge and skills needed to minimize the losses to their agricultural production caused by hailstorms, as well as low resources to tackle the risks. All the farmers expressed the opinion that they did not perceive the existence of any policy to compensate them after the occurrence of hailstorms; thus, they did not receive any incentives when hailstorms caused significant damage to their crop production. In total, $64 \%$ of respondents felt at risk from hailstorms.

\section{Farmers' Hailstorm Risk Perceptions}

The findings on farmers' hailstorm risk perceptions indicated that, of the $64 \%$ of total respondents who perceived hailstorm risk, about $98 \%$ had perceived the risk, whereas $2 \%$ were unsure of the extent to which they had perceived the risk. On the other hand, of the $90 \%$ of farmer respondents with a highly perceived hailstorm risk, $2 \%$ perceived hailstorms as being less of a risk. Moreover, $6 \%$ of farmers had a moderately perceived hailstorm risk to their crop production and agricultural activities (Fig. 3).

\section{Estimation of Multicollinearity between the Variables}

The relationships between the dependent variable and the independent variables and between the independent variables were examined using the Pearson correlation coefficient test. Considering the dependent variable, that is, hailstorm risk perceptions, we found significant positive correlations with respondents' level 
Table 1. Descriptive analysis of variables employed in the study.

\begin{tabular}{|c|c|c|}
\hline Variables & Description & Mean \& SD \\
\hline \multicolumn{3}{|c|}{ Independent variables (household's socio-demographic and economic characteristics) } \\
\hline $\mathrm{V}_{1} \cdot$ Age (years) & Continuous & $49.90 \pm 13.48$ \\
\hline $\mathrm{V}_{2}$. Level of education (years of schooling) & Continuous & $6.59 \pm 3.62$ \\
\hline $\mathrm{V}_{3}$. Farm size (hectare $\left.[\mathrm{ha}]\right)$ & Dummy receives the value of 1 if a large farm ( $>1.0$ ha), 0 otherwise & $0.67 \pm 0.44$ \\
\hline $\mathrm{V}_{4}$. Predecessor farm size (ha) & Dummy receives the value of 1 if a large farm ( $>1.0$ ha), 0 otherwise & $2.66 \pm 1.18$ \\
\hline $\mathrm{V}_{5}$. Annual family income (USD ‘000) & $\begin{array}{l}\text { Dummy receives the value of } 1 \text { if a high income (> USD 1,500), } \\
\qquad 0 \text { otherwise }\end{array}$ & $1.30 \pm 0.22$ \\
\hline $\mathrm{V}_{6}$. Household assets (USD ‘000) & Continuous & $1.45 \pm 0.50$ \\
\hline $\mathrm{V}_{7}$. Agricultural training experience (days) & Continuous & $3.69 \pm 2.68$ \\
\hline $\mathrm{V}_{8} \cdot$ Credit received (USD ‘000) & Continuous & $0.24 \pm 0.10$ \\
\hline $\begin{array}{l}\mathrm{V}_{9} . \text { Extension media contact (total contact } \\
\text { score) }\end{array}$ & $\begin{array}{l}\text { Dummy receives the value of } 1 \text { if high level of contact }(>50 \%) \text {, } \\
\qquad 0 \text { otherwise }\end{array}$ & $12.34 \pm 5.41$ \\
\hline $\begin{array}{l}\mathrm{V}_{10} . \text { Organizational participation (total } \\
\text { participation score) }\end{array}$ & $\begin{array}{l}\text { Dummy receives the value of } 1 \text { if high participation }(>50 \%) \text {, } \\
\qquad 0 \text { otherwise }\end{array}$ & $8.25 \pm 3.18$ \\
\hline \multicolumn{3}{|c|}{ Objective risk factors } \\
\hline $\mathrm{V}_{11}$. Exposure to hailstorms & Dummy receives the value of 1 if high probability, and 0 otherwise & $0.94 \pm 0.23$ \\
\hline $\mathrm{V}_{12}$. Sensitivity to hailstorms & Dummy receives the value of 1 if high impact, and 0 otherwise & $0.95 \pm 0.30$ \\
\hline \multicolumn{3}{|c|}{ Subjective risk factors } \\
\hline $\mathrm{V}_{13}$. Adaptation efficacy & $\begin{array}{c}\text { Dummy receives the value of } 1 \text { if high adaptation efficacy, and } 0 \\
\text { otherwise }\end{array}$ & $0.47 \pm 0.50$ \\
\hline $\mathrm{V}_{14} \cdot$ Self-efficacy & Dummy receives the value of 1 if high self-efficacy, and 0 otherwise & $0.67 \pm 0.47$ \\
\hline $\mathrm{V}_{15}$. Adaptation cost-carrying capability & $\begin{array}{l}\text { Dummy receives the value of } 1 \text { if high adaptation cost-carrying capa- } \\
\text { bility, and } 0 \text { otherwise }\end{array}$ & $0.45 \pm 0.50$ \\
\hline \multicolumn{3}{|c|}{ Policy factors } \\
\hline $\begin{array}{l}\mathrm{V}_{16} . \text { Policy to provide any incentives after } \\
\text { hailstorm occurrences }\end{array}$ & $\begin{array}{c}\text { Binary receives the value of } 1 \text { if any policy is perceived, } \\
\text { and } 0 \text { otherwise }\end{array}$ & $0.00 \pm 0.00$ \\
\hline \multicolumn{3}{|c|}{ Dependent variable (Hailstorm risk perceptions) } \\
\hline $\begin{array}{c}\mathrm{V}_{0} \text {. Any type of feelings of risk due to } \\
\text { hailstorms }\end{array}$ & Binary receives the value of 1 if any feelings of risk, and 0 otherwise & $0.64 \pm 0.48$ \\
\hline
\end{tabular}

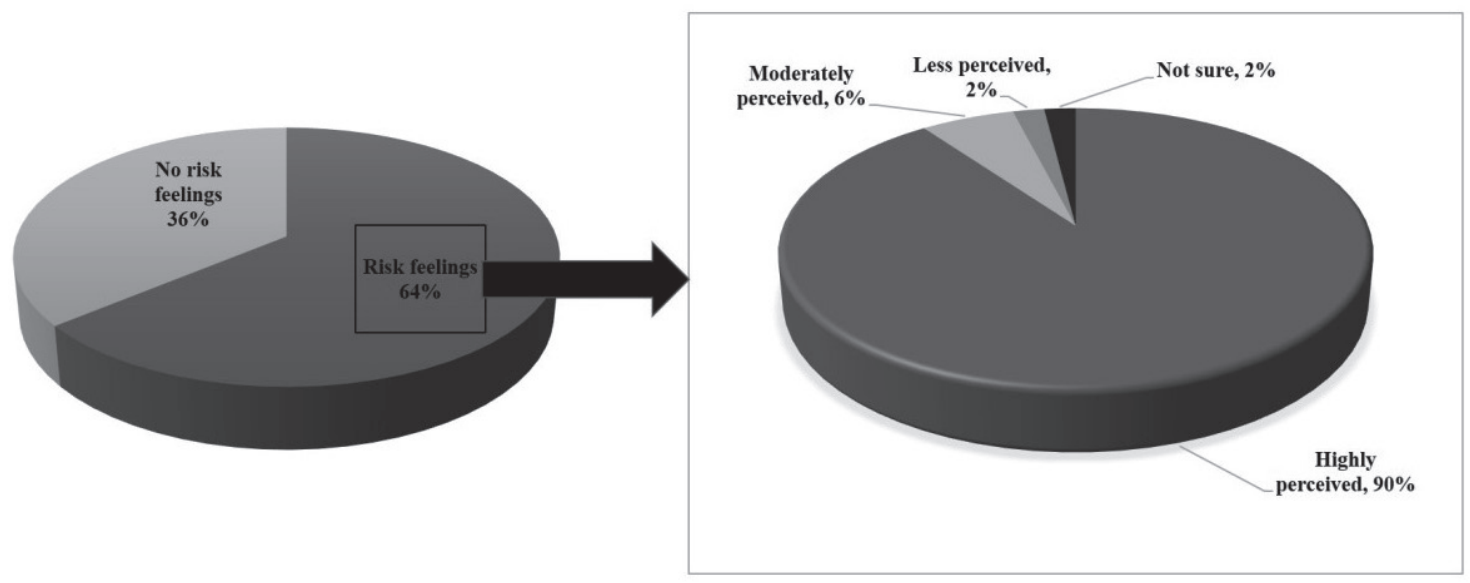

Fig. 3. Hailstorm risk perceptions of farmers in Panchagarh district. 


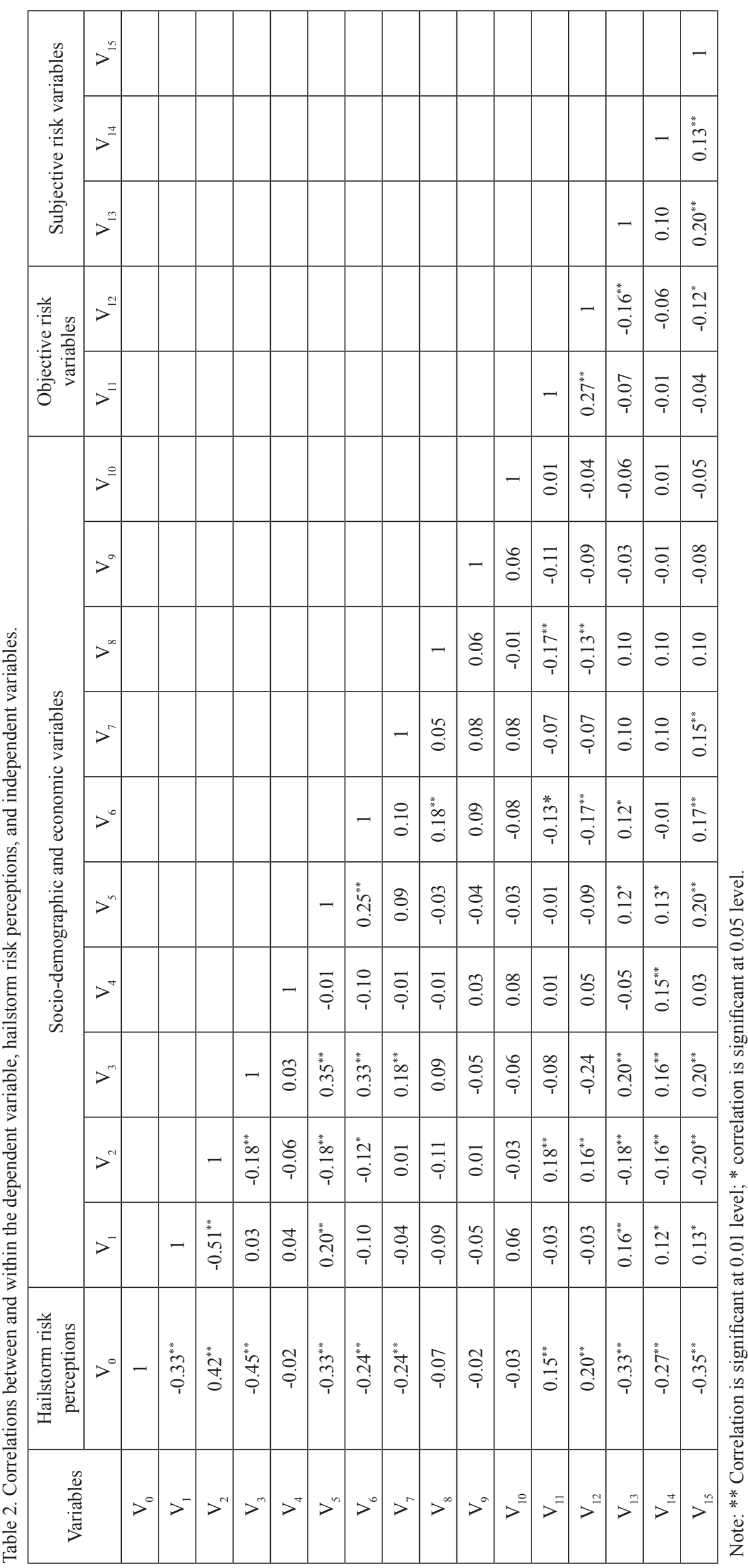


of education, exposure, and sensitivity. On the other hand, we found strong negative correlations with their age, farm size, annual household income, household assets, agricultural training, adaptation efficacy, selfefficacy, and adaptation cost. A strong correlation was observed between age and education; age and annual family income; age and adaptive capacity; education and household income; education and perceived impact; education and adaptive capacity; farm size and annual household income; farm size and adaptive capacity; exposure and sensitivity; sensitivity and adaptive capacity; and exposure and adaptive capacity. None of the correlation coefficient values were more than 0.7 . However, the correlations expected for all the results are shown in Table 2. Even though correlations were found between some variables, due to the study purpose, all variables showing correlations were taken into the binary logistic regression model.

\section{Determinants of Hailstorm Risk Perceptions}

Binary logistic regression was applied to assess the contribution of several factors, such as perceived risk factors, adaptive capacity, and socio-demographic and economic variables on respondents who felt extreme risk. The model contained 15 independent variables. The full model containing all predictor variables was statistically significant, $\chi^{2}(15, \mathrm{~N}=320)=160.82$, $p<0.001$, indicating that it could differentiate between respondents who reported risk feelings towards hailstorms and those who did not report such feelings. Overall, the model explained between 39.5\% (Cox and Snell R-squared) and 54.0\% (Nagelkerke R-squared) of the variance in risk perceptions, and correctly classified $84.4 \%$ of cases. The sensitivity of the model could be expressed as $90.6 \%$ of correct predictions in the category of interests on risk perceptions. The specificity of the model could also be revealed as $73.5 \%$ of the true negative. The model fitted well based on Hosmer and Lemeshow's test (the goodness-of-fit score was 0.56 which was much higher than the recommended cut-off value of 0.05 ).

As shown in Table 3, of the 15 independent variables, only eight (i.e., age, level of education, farm size, annual income, agricultural training experience, adaptation efficacy, self-efficacy, and adaptation cost) made a unique statistically significant contribution to the model. The following paragraph presents the factors that influence hailstorm risk perception.

Respondents' age contributes to this model, with this expressed as the higher the age, the lower the risk perception. Young and middle-aged farmers reported higher risk than was the case with farmers who were older. The correlation was negative (statistically

Table 3. Estimates of binary logit regression model for hailstorm risk perceptions.

\begin{tabular}{|c|c|c|c|c|c|c|c|c|}
\hline \multirow{2}{*}{ Factors } & \multirow{2}{*}{$\mathrm{B}$} & \multirow{2}{*}{$\mathrm{SE}$} & \multirow{2}{*}{ Wald } & \multirow{2}{*}{$d f$} & \multirow{2}{*}{ Sig. } & \multirow{2}{*}{ OR } & \multicolumn{2}{|c|}{$95 \% \mathrm{CI}$ for OR } \\
\hline & & & & & & & Lower & Upper \\
\hline \multicolumn{9}{|c|}{ Socio-demographic and economic variables } \\
\hline Age & -0.04 & 0.02 & 6.31 & 1 & 0.01 & 0.96 & 0.94 & 0.99 \\
\hline Level of education & 0.22 & 0.06 & 15.44 & 1 & 0.00 & 1.25 & 1.12 & 1.39 \\
\hline Farm size & 0.97 & 0.37 & 7.23 & 1 & 0.00 & 2.68 & 1.31 & 5.50 \\
\hline Annual income & 0.93 & 0.36 & 6.78 & 1 & 0.00 & 2.52 & 1.26 & 5.06 \\
\hline Agricultural training & -0.24 & 0.06 & 15.28 & 1 & 0.00 & 0.79 & 0.70 & 0.89 \\
\hline \multicolumn{9}{|c|}{ Subjective risk variables } \\
\hline Adaptation efficacy & 1.11 & 0.32 & 12.30 & 1 & 0.00 & 3.04 & 1.63 & 5.65 \\
\hline Self-efficacy & 1.03 & 0.36 & 8.08 & 1 & 0.00 & 2.80 & 1.38 & 5.69 \\
\hline Adaptation cost & 1.18 & 0.32 & 13.95 & 1 & 0.00 & 3.26 & 1.75 & 6.06 \\
\hline Constant & 0.72 & 1.08 & 0.45 & 1 & 0.50 & 0.49 & & \\
\hline$-2 \log$ likelihood & \multicolumn{8}{|c|}{259.40} \\
\hline Cox \& Snell R square, & \multicolumn{8}{|c|}{$39.5 \%$} \\
\hline Nagelkerke R square & \multicolumn{8}{|c|}{$54 \%$} \\
\hline Hosmer and Lemeshow & \multicolumn{8}{|c|}{$\chi^{2}=6.67, d f=8, p=0.56$} \\
\hline
\end{tabular}

Notes: -B: unstandardized regression weight; SE: standard error; OR: odds ratio; Wald: Wald chi-square value; $d f$ : degrees of freedom, Sig.: significance; CI: confidence interval. 
significant at $1 \%$ level) and lay between age and level of education, with this meaning that the higher the age, the lower the level of education (Table 2). The odds ratio (OR) for age was 0.96 , with this indicating that, with every unit of increase in age, the risk perception decreased 0.96 times. The odds ratio (OR) for the level of education variable at 1.25 indicated that, for each unit of increase in the level of education, the risk perception increased 1.25 times. Farm size was the strongest socio-economic predictor for reporting an extreme feeling of risk, recording an odds ratio (OR) of 2.68. This indicated that smallholder farmer respondents were 2.68 times more likely to report a feeling of risk than medium-sized and large farm-holder farmer respondents, controlling for all other factors in the model. Annual income significantly contributed to the model, revealing that the farmers with a lower annual income reported 2.52 times more risk than the farmers with a higher income. Agricultural training is very important for farmers in Bangladesh as a significant proportion of farmers are illiterate or have only primary education; therefore, continuous on-farm as well as off-farm training from the agricultural department to improve farming and to adapt to the changing climate are essential. From Table 3, we can see that, for every unit of increase in agricultural training, the risk perception decreased 0.79 times.

Overall, the subjective risk factors comprising the adaptive capacity of the farmers most significantly contributed to the model. The farmers who perceived that they had lower adaptation efficacy reported 3.03 times more risk than the farmers who perceived that they had medium to higher adaptation efficacy. Moreover, the farmers who perceived that they had lower selfefficacy, meaning a lower level of knowledge and skills, reported 2.80 times more risk than the farmers who perceived that they had higher self-efficacy. The resources to tackle the hazard (i.e., the adaptation cost) play the most important role in adaptive capacity and the timeliness of decision making to adopt different adaptation strategies. The adaptation cost-carrying capability most significantly contributed to the model and acted as the strongest predictor variable among the other variables. The farmers who perceived that they had lower resources to tackle the risks of hailstorms reported 3.26 times more risk than the farmers who perceived that they had higher adaptation cost-carrying capability.

\section{Discussion}

This study has analyzed factors influencing farmers' hailstorm risk perceptions. The study partially adopted the conceptual framework developed by Duinen et al. (2015) [7] and added a new dimension; that is, policy factors were taken into consideration for the study. The determinants for farmers' risk feelings towards hailstorms, in particular, have not been explored in previous studies. Therefore, it is difficult to compare the current study's findings with the significant amount of other hailstorm risk perception-related research conducted globally as well as in Bangladesh. However, the current study's results provide several ideas about determinants and their level of contribution to hailstorm risk perceptions of farmers. Farmers' hailstorm risk perceptions reflect actual hailstorm risk exposure and sensitivity, as measured by Raihan et al. (2020) [13].

Many empirical studies can be found that have assessed the determinants of different natural hazards, but this is the very first study to link objective and subjective hailstorm risk factors and farmers' risk perceptions. In the prior literature, Duinen et al. (2015) made a link between objective and subjective risk factors of drought risk perceptions [7]. The current study's findings using bivariate correlations provide evidence that farmers who perceived more probability of the occurrence of hailstorms perceived greater risks. Furthermore, the findings also indicate that the farmers who were objectively more vulnerable to hailstorms, which would have higher impact on their farms, perceived higher risk. Though the empirical model didn't demonstrate the explanatory power of the objective risk factors, still, those are important as determinants of hailstorm risk perceptions. These findings were in line with those of Duinen et al. (2015) who found that the farmers who perceived more exposure and sensitivity to drought perceived the risks to be higher [7]. Habiba et al. (2012) [52] and Tang et al. (2013) [53] had similar findings, revealing that natural hazard-related objective factors perceived by farmers had a significant influence on their risk perceptions.

On the other hand, the significant contribution of several subjective risk factors to the explanation of farmers' hailstorm risk perceptions provides an insight that their risk perceptions differ from the actual objective hailstorm risk due to differences in farmers' personal circumstances. Below et al. (2012) found that the risk feelings of farmers towards drought and floods were determined by their socio-economic characteristics, particularly age, education, income, knowledge, etc. [54]. We found that farm size or a farmer having his/her own land, age, level of education, annual income, and agricultural training were important determinants. Alam (2014) found that the vulnerability to, and risks of, drought were determined by farmers' economic conditions and the size of the farms owned by them [55]. Halkos (2019) demonstrated that small and medium sized entrepreneurs are disproportionately affected by EWEs [56], and Skouloudis et al. (2020) found that small and medium-sized enterprises are particularly vulnerable to EWEs which is similar to the present study that the smallholder farmers face greater vulnerability to their farms, thus, greater risks [57]. Marginal farmers as well as smallholders typically have less options for managing the risks of hailstorms [19]. When hailstorms occur and their crops are damaged, they do not have the option of minimizing their crop 
losses as the land size is smaller and hailstorms are localized hazards. On the other hand, farmers who own medium-sized to large farms can, to some extent, manage the losses if some of their land is not affected or is less affected.

In the current study, the young and middle-aged farmers had higher levels of education and, thus, they were more concerned about risk factors and the existing policy for hailstorms. Shijin and Dahe (2015) conducted research on mountain farmers' risk perceptions towards natural hazards and found that those who were younger felt more risks than those who were older, a similar finding to findings in the current study [58]. Moreover, the younger farmers reported their loss of motivation for continuing to farm. It is possible that the older farmers might consider the issue of hailstorm occurrence and hailstorms' greater impact on their farms as being common, and that they had to adapt to such situations and continue their crop production. This might be a possible reason for risk perception differences as farmers' ages increased. The younger farmers perceived more risks, with these having very negative consequences closely linked to sustainable agriculture. Several other studies have found significant relationships between age and risk perceptions [52, 59]. Raihan et al. (2020) found that more than $30 \%$ of the farmers could lose their motivation for farming as a result of the rapid emergence and increasing risks of hailstorms in northern Bangladesh. This is a significant issue for sustainable agriculture [13]. Bryan (2009) found that younger farmers perceived more risks of drought occurrence than was the case with older farmers [38]. One possible reason might be their farming experience and knowledge of long-term farming and climatic variables.

This study found that access to agricultural training generally leads to increasing farmers' adaptive capacity and, most importantly, increasing their self-efficacy for minimizing losses caused by hailstorms [18]. Thus, agricultural training acts as a determinant of farmers' hailstorm risk perceptions. Therefore, it can be predicted that the provision of more training can increase farmers' ability to cope with adverse situations caused by hailstorms and, eventually, that farmers' feelings of risk might decrease. This is in line with Zakaria et al. (2020) who found that farmers' participation in agricultural training acted as a significant determinant for climate change adaptation practices, ultimately leading to risk perceptions towards climate-induced hazards [39].

Moreover, it seems that subjective factors related to psychological variables are more substantial risk perception factors than socio-demographic and economic variables such as age, education, annual income, etc. This is the most important finding of the current study, indicating that the influence of psychological factors on risk perception is the most significant and powerful determinant. Farmers perceived that they had a low level of ability to tackle hailstorm risk; low knowledge and skills for managing losses caused by hailstorms; and, most importantly, 'low' capability for bearing the adaptation cost to manage hailstorm risks. However, successful adoption of the best-suited adaptation strategies would depend on farmers' self-efficacy and adaptation cost-carrying capability $[18,60]$. Some probable reasons for the above perceptions of farmers could be fewer resources, smaller farm size, continuing land fragmentation, installation of factories and tea industries in the study area (i.e., selling their land to large companies); shortage of liquid money to manage adaptation strategies; temporary vulnerability to poverty; and indifferent attitudes to risk management. Many researchers have found that farmers with less adaptive capacity perceived more risks. Islam et al. (2020) [19], Habiba (2012) [52], Ajzen (2002) [42], Slovic et al. (2004) [61], and Barnes et al. (2013) [62] had similar results, indicating that subjective psychological variables related to the perceived ability or adaptive capacity to minimize impacts have a significant contribution to risk perceptions and acted as the most important determinants of risk perceptions.

The current study's conceptual framework (Fig. 1) stated that objective and subjective risk factors might make a significant contribution to farmers' hailstorm risk perceptions, with the study finding that subjective factors were significant determinants. The objective risk factors, namely, exposure and sensitivity, could not contribute to the empirical model as both these variables were not normally distributed and about $95 \%$ of farmers expressed the same views about higher exposure and sensitivity. But it can be assumed that farmers felt risk to hailstorms due to their higher exposure and sensitivity. The objective risk factors are the recessive factors of risk perception. Moreover, as no compensation policy was in place for implementation after a severe hailstorm occurrence, the policy factor served as a recessive factor which was significant regardless of whether or not farmers perceived the risks of hailstorms. Raihan et al. (2020) found that the policy factor played the most important role in increasing community risk perceptions of hailstorms [13]. Finally, the current study argues that subjective risk perception factors hold their interpretive power in a hailstorm risk perception model, and that farmers depend on their long-term farming experience to shape their hailstorm risk assessment in a rational way. Moreover, the adaptive capacity of farmers is the main concern in relation to their hailstorm risk perceptions; thus, appropriate measures to enhance their adaptive capacity could be helpful to minimize the hailstorm risks faced by farming communities.

\section{Conclusion}

This study's findings reflect the perceptions of farmers in selected areas of northern Bangladesh and have wider implications for natural hazardprone developing countries, particularly those prone 
to hailstorms and based on agriculture. This study is crucial considering the recent increasing trend of hailstorms and vulnerability to the agriculture sector of Bangladesh. Moreover, this is the first attempt of exploring the determinants of hailstorm risk perceptions, which have several practical implications to the concerned sectors. The findings show that personal circumstances and socio-economic characteristics made a significant contribution to farmers' risk perceptions. One of the important findings of this study is the contribution of age and level of education to the risk perception where young farmers who have comparatively higher education are perceiving greater risks, which have potential implications for policy planners and most importantly extension agents. If the young farmers perceive more risks and lose the motivation of farming, it will produce a greater threat to sustainable agriculture. So, necessary steps should be taken to reduce the risk feelings of the young farmers, particularly by compensating for the losses of hailstorms in agricultural sectors. More agricultural training for effective adaptation measures can enhance the adaptive capacity of farmers, thus, reduce the risk feelings. Moreover, subjective factors related to adaptive capacity were the most significant factors in farmers' risk perceptions. Farmers depended on their personal experiences for objective measurement as well as their adaptive capacity to judge hailstorm risk; thus, subjective factors played an important role in hailstorm risk perceptions.

These results have implications for policy makers in the formulation of the hailstorm risk management policy. Moreover, the adaptive capacity of farmers against hailstorms should be enhanced. Like all other EWEs and natural disasters, a compensation policy, incentives or the availability of crop insurance after hailstorm occurrences could be helpful in reducing farmers' feelings of risk. This study recommends that a continuous capacity development program should be implemented to improve the adaptive capacity of farmers by covering larger training facilities, establishing structural and non-structural risk reduction measures of hailstorms, and providing logistic support to adopt appropriate adaptation practices. In relation to further research, it is essential to know the extent of hailstorm risk, as estimated by concerned experts, so this can be compared with farmers' perceptions. If farmers appear to have unsubstantiated and unreal hailstorm risk perceptions, this could ultimately affect their adaptation behavior, and the sustainability of their agriculture would be questioned. Subsequent research could also elaborate on farmers' expectations for incentives, crop insurance or a compensation policy after a severe hailstorm occurrence, thus seeking to minimize their perceptions of hailstorm risk. Similarly, further investigation could be conducted on the possible role of providing adaptation costs, increasing adaptation skills and knowledge through training and, overall, increasing farmers' adaptation efficacy to minimize their risk. In terms of replicability, future studies can refer to the approach of this study. Required information may include the data on subjective and objective risk factors, socio-demographic and economic factors including the social and policy influences for the particular natural hazard. However, the result may differ due to the perception-based approach this study is using. If there are enough number of cases, there is a strong possibility to do a comparative study (crossregion, cross countries, etc.). Also, there is another possibility to implement a similar approach in other sectors, for example, business and natural disaster risk reduction and management. To do so, those studies should be developed on perception-centred topics. The important limitation of this study lies in its assessment of hailstorm risk perceptions. This research used crosssectional survey data at a given time and at a given location; however, risk perceptions are not static but are instead dynamic and can differ with time and location.

\section{Acknowledgments}

The first author is grateful to the Ministry of Education, Sports, Science, and Technology (MEXT) of Japan for financial support and civil administration, agriculture department, and to the local people of Panchagarh, Bangladesh, for their cooperation and support in conducting field studies. The first author also expresses his deep gratitude to Professor Dr. M Zulfikar Rahman and Professor Dr. Asaduzzaman Sarker, Department of Agricultural Extension Education, Bangladesh Agricultural University, Bangladesh, for their scholastic directions in designing the study questionnaire.

\section{Conflict of Interest}

The authors declare no conflict of interest.

\section{References}

1. PlANTON S., DEQUE M., CHAUVIN F., TERRAY L. Expected impacts of climate change on extreme climate events. Extern. Geophys. Clim. Environ. 340, 564, 2008.

2. SARKER M.N.I., YANG B., LV Y., HUQ M.E., KAMRUZZAMAN M.M. Climate change adaptation and resilience through big data. Int. J. Adv. Comput. Sci. Appl. 11, 533, 2020.

3. MUNICH R.E. Munich Reinsurance Company Geo Risks Research - Annual Report 2012. 148, 2013.

4. SAHA T.R., QUADIR D.A. Variability and trends of annual and seasonal thunderstorm frequency over Bangladesh. Int. J. Climatol. 36, 4651, 2016.

5. CECIL D.J., BLANKENSHIP C.B. Toward a Global Climatology of Severe Hailstorms as Estimated by Satellite Passive Microwave Imagers. J. Clim. 25, 687, 2012.

6. HALKOS G., SKOULOUDIS A., MALESIOS C., JONES $\mathrm{N}$. A hierarchical multilevel approach in assessing factors 
explaining country-level climate change vulnerability. Sustain. 12, 2020.

7. DUINEN R.V., FILATOVA T., GEURTS P., VEEN A.V.D. Empirical Analysis of Farmers' Drought Risk Perception: Objective Factors, Personal Circumstances, and Social Influence. Risk Anal. 35, 741, 2015.

8. SARKER M.N.I., WU M., ALAM G.M.M., SHOUSE R.C. Life in riverine islands in Bangladesh: Local adaptation strategies of climate vulnerable riverine island dwellers for livelihood resilience. Land use policy 94, 104574, 2020.

9. HALKOS G., SKOULOUDIS A., MALESIOS C., EVANGELINOS K. Bouncing Back from Extreme Weather Events: Some Preliminary Findings on Resilience Barriers Facing Small and Medium-Sized Enterprises. Bus. Strateg. Environ. 27, 547, 2018.

10. MIRZA M.M.Q. Climate change and extreme weather events: can developing countries adapt? Climate change and extreme weather events: can developing countries adapt? Clim. Policy 3, 233, 2011.

11. WEBB J.D.C., ELSOM D.M., REYNOLDS D.J. Climatology of severe hailstorms in Great Britain. Atmos. Res. 56, 291, 2001.

12. ZHENG Y., BYG A. Coping with climate change: Households' response strategies to drought and hailstorm in Lijiang, China. Environ. Hazards 13, 211, 2014.

13. RAIHAN L., ONITSUKA K., BASU M., SHIMIZU N., HOSHINO S. Rapid Emergence and Increasing Risks of Hailstorms: A Potential Threat to Sustainable Agriculture in Northern Bangladesh. Sustainability 12, 5011, 2020.

14. BUSHRA N., TREPANIER J.C., ROHLI R. V. Joint probability risk modelling of storm surge and cyclone wind along the coast of Bay of Bengal using a statistical copula. Int. J. Climatol. 39, 4206, 2019.

15. ALAM G.M.M., ALAM K., MUSHTAQ S., SARKER, M.N.I., HOSSAIN M. Hazards, food insecurity and human displacement in rural riverine Bangladesh: Implications for policy. Int. J. Disaster Risk Reduct. 43, 101364, 2020.

16. Government of the People's Republic of Bangladesh. Ministry of Disaster Management and Relief National Plan for Disaster Management 2016-2020; 2017.

17. RAHMAN A.A., ALAM M., ALAM S.S., UZZAMAN M.R., RASHID M., RABBANI G. UNDP human development report 2007: background paper on risks, vulnerability and adaptation in Bangladesh; 2008.

18. SARKER M.N.I., WU M., ALAM G.M.M., SHOUSE, R.C. Administrative resilience in the face of natural disasters: Empirical evidence from Bangladesh. Pol. J. Environ. Stud. 29, 1825, 2020.

19. ISLAM M.D.I., RAHMAN A., SARKER M.N.I., SARKER M.S.R., JIANCHAO L. Factors influencing rice farmers' risk attitudes and perceptions in bangladesh amid environmental and climatic issues. Pol. J. Environ. Stud. 30, 177, 2021.

20. SULEWSKI P., KLOCZKO-GAJEWSKA A. Farmers' risk perception, risk aversion and strategies to cope with production risk: an empirical study from Poland. Stud. Agric. Econ. 116, 140, 2014.

21. GOULDSON A., MORTON A., POLLARD S.J.T. Better environmental regulation - contributions from riskbased decision-making. Sci. Total Environ. 407, 5283, 2009.

22. WACHINGER G., RENN O. Risk perception of natural hazards. WP3-Report of the 1, 2010.

23. YANG J., QIU W. A measure of risk and a decisionmaking model based on expected utility and entropy. Eur. J. Oper. Res. 164, 792, 2005.
24. BOTTERILL B.L., MAZUR N., MAZUR N. Risk and Risk perception. Nature 361, 689, 1993.

25. TERPSTRA T., LINDELL M.K., GUTTELING J.M. Does communicating (flood) risk affect (flood) risk perceptions? results of a quasi-experimental study. Risk Anal. 29, 1141, 2009.

26. FERNANDEZ G., TUN A.M., OKAZAKI K., ZAW S.H., KYAW K. Factors influencing fire, earthquake, and cyclone risk perception in Yangon, Myanmar. Int. J. Disaster Risk Reduct. 28, 140, 2018.

27. AHAMMED S., CHUNG E.-S., SHAHID S. Parametric Assessment of Pre-Monsoon Agricultural Water Scarcity in Bangladesh. Sustainability 10, 819, 2018.

28. HALKOS G.E., TSIRIVIS A.S. Value-at-risk methodologies for effective energy portfolio risk management. Econ. Anal. Policy 62, 197, 2019 doi:10.1016/j.eap.2019.03.002.

29. NOSIC A., WEBER, M. Changes of expectations and risk attitudes and their impact on risk taking behavior. SSRN eLibrary 2009.

30. SJOBERG L. Factors in risk perception. Risk Anal. 20, 1, 2000.

31. JANMAIMOOL P., WATANABE T. Evaluating determinants of environmental risk perception for risk management in contaminated sites. Int. J. Environ. Res. Public Health 11, 6291, 2014.

32. FISCHHOFF B., WATSON S.R., HOPE C. Defining risk. Policy Sci. 17, 123, 1984.

33. SAQIB S.E., AHMAD M.M., PANEZAI S., RANA I.A. An empirical assessment of farmers' risk attitudes in flood-prone areas of Pakistan. Int. J. Disaster Risk Reduct. 18, 107, 2016.

34. PATT A.G., SCHROTER D. Perceptions of climate risk in Mozambique: Implications for the success of adaptation strategies. Glob. Environ. Chang. 18, 458, 2008.

35. ROEHL W.S., FESENMAIER D.R. Risk Perceptions and Pleasure Travel: An Exploratory Analysis. J. Travel Res. 30, 17, 1992.

36. KEIL M., WALLACE L., TURK D., DIXON-RANDALL G., NULDEN U. Investigation of risk perception and risk propensity on the decision to continue a software development project. J. Syst. Softw. 53, 145, 2000.

37. HASAN M.K., KUMAR L. Comparison between meteorological data and farmer perceptions of climate change and vulnerability in relation to adaptation. J. Environ. Manage. 237, 54, 2019.

38. BRYAN E., DERESSA T.T., GBETIBOUO G.A., RINGLER C. Adaptation to climate change in Ethiopia and South Africa: options and constraints. Environ. Sci. Policy 12, 413, 2009.

39. ZAKARIA A., AZUMAH S.B., APPIAH-TWUMASI M., DAGUNGA G. Adoption of climate-smart agricultural practices among farm households in Ghana: The role of farmer participation in training programmes. Technol. Soc. 63, 101338, 2020.

40. SLOVIC P., FINUCANE M., PETERS E., MACGREGOR D.G. Risk as feeling: Some thoughts about affect, reason, risk and rationality. Ethics Technol. Risk 24, 163, 2004.

41. NDAMANI F., WATANABE T. Determinants of farmers' climate risk perceptions in agriculture-a rural ghana perspective. Water (Switzerland). 9, 2017.

42. AJZEN I. Perceived behavioral control, self-efficacy, locus of control, and the theory of planned behavior. J. Appl. Soc. Psychol. 32, 665, 2002. 
43. AHSAN D.A. Farmers' motivations, risk perceptions and risk management strategies in a developing economy: Bangladesh experience. J. Risk Res. 14, 325, 2011.

44. SLOVIC P., FISCHHOFF B., LICHTENSTEIN S. Why Study Risk Perception? Risk Anal. 2, 83, 1982.

45. SIOUTAS M., MEADEN T., WEBB J.D.C. Hail frequency, distribution and intensity in Northern Greece. Atmos. Res. 93, 526, 2009.

46. PANCHAGARH UPAZILA NIRDESHIKA. In Bangladesh Soil Resources Development institute (SRDI). Dhaka, Bangladesh; p. Series-411, 2019.

47. RAIHAN M., SARKER M., MIAH, M. Shortage of water in Teesta river basin and its impact on crop production in northern Bangladesh. SAARC J. Agric. 15, 113, 2018.

48. BROCKWELL P.J., DAVIS R.A. Springer Series in Statistics Advisors:; ISBN 1-4419-0319-8, 2006.

49. UDDIN M.N., BOKELMANN W., ENTSMINGER J.S. Factors affecting farmers' adaptation strategies to environmental degradation and climate change effects: A farm level study in bangladesh. Climate 2, 223, 2014.

50. QUIROGA S., SUAREZ C., SOLIS J.D. Exploring coffee farmers' awareness about climate change and water needs: Smallholders' perceptions of adaptive capacity. Environ. Sci. Policy 45, 53, 2015.

51. JAE JEONG H. The level of collapse we are allowed: Comparison of different response scales in Safety Attitudes Questionnaire. Biometrics Biostat. Int. J. 4, 128, 2016.

52. HABIBA U., SHAW R., TAKEUCHI Y. Farmer's perception and adaptation practices to cope with drought: Perspectives from Northwestern Bangladesh. Int. J. Disaster Risk Reduct. 1, 72, 2012.

53. TANG J., FOLMER H., XUE J. Estimation of awareness and perception of water scarcity among farmers in the Guanzhong Plain, China, by means of a structural equation model. J. Environ. Manage. 126, 55, 2013.
54. BELOW T.B., MUTABAZI K.D., KIRSCHKEL D., FRANKE C., SIEBER S., SIEBERT R., TSCHERNING $\mathrm{K}$. Can farmers' adaptation to climate change be explained by socio-economic household-level variables? Glob. Environ. Chang. 22, 223, 2012.

55. ALAM G.M.M., ALAM K., MUSHTAQ S. Climate change perceptions and local adaptation strategies of hazard-prone rural households in Bangladesh. Clim. Risk Manag. 17, 52, 2017.

56. HALKOS G., SKOULOUDIS A. Investigating resilience barriers of small and medium-sized enterprises to flash floods: a quantile regression of determining factors. Clim. Dev. 12, 57, 2020.

57. SKOULOUDIS A., TSALIS T., NIKOLAOU I., EVANGELINOS K., FILHO W.L. Small \& medium-sized enterprises, organizational resilience capacity and flash floods: Insights from a literature review. Sustain. 2020, 12, doi:10.3390/SU12187437.

58. SHIJIN W., DAHE Q. Mountain inhabitants' perspectives on climate change, and its impacts and adaptation based on temporal and spatial characteristics analysis: a case study of Mt. Yulong Snow, Southeastern Tibetan Plateau. Environ. Hazards 14, 122, 2015.

59. SLEGERS M.F.W. If only it would rain: Farmers' perceptions of rainfall and drought in semi-arid central Tanzania. J. Arid Environ. 72, 2106, 2008.

60. ALI M.F., ASHFAQ M., HASSAN S., ULLAH R. Assessing Indigenous Knowledge through Farmers' Perception and Adaptation to Climate Change in Pakistan. Pol. J. Environ. Stud. 27, Vol. 29; 2018.

61. SLOVIC P., FINUCANE M., PETERS E., MACGREGOR D.G. Risk as feeling: Some thoughts about affect, reason, risk and rationality. Ethics Technol. Risk 24, 163, 2012.

62. BARNES A.P.,, ISLAM M.M., TOMA L. Heterogeneity in climate change risk perception amongst dairy farmers: A latent class clustering analysis. Appl. Geogr. 41, 105, 2013. 